\title{
Intravoxel incoherent motion MRI: emerging applications for nasopharyngeal carcinoma at the primary site
}

\author{
Shui-xing Zhang $\cdot$ Qian-jun Jia $\cdot$ Zhong-ping Zhang • \\ Chang-hong Liang • Wen-bo Chen • Qian-hui Qiu $\cdot$ He Li
}

Received: 12 November 2013 / Revised: 14 March 2014 / Accepted: 24 April 2014 / Published online: 18 May 2014

(C) The Author(s) 2014. This article is published with open access at Springerlink.com

\begin{abstract}
Objectives We compared pure molecular diffusion $(D)$, perfusion-related diffusion $\left(D^{*}\right)$, perfusion fraction $(f)$ and apparent diffusion coefficient (ADC) based on intravoxel incoherent motion (IVIM) theory in patients with nasopharyngeal carcinoma (NPC).

Methods Sixty-five consecutive patients (48 men) with suspected NPC were examined using a 3.0-T MR system. Diffusion-weighted imaging (DWI) was performed with 13 $b$ values (range, $0-800 \mathrm{~s} / \mathrm{mm}^{2}$ ). We regarded the result of endoscopy and biopsy as the gold standard for detection. $D$, $D^{*}$ and $f$ were compared between patients with primary NPC and enlarged adenoids.

Results IVIM DWI was successful in 37 of 40 NPC and 23 of 25 enlarged adenoids cases. $D(P=0.001)$ and $f(P<0.0001)$ were significantly lower in patients with NPC than in patients with enlarged adenoids, whereas $D^{*}$ was significantly higher $(P<0.0001)$. However, the ADC was not significantly different between the two groups $(P>0.05)$. The area under the ROC curve (AUC) for $D$ was 0.849 and was significantly larger than that for ADC $(P<0.05)$.

Conclusions IVIM DWI is a feasible technique for investigating primary NPC. $D$ was significantly decreased in primary NPC, and increased $D^{*}$ reflected increased blood vessel generation and parenchymal perfusion in primary NPC.
\end{abstract}

S.-x. Zhang • Q.-j. Jia • C.-h. Liang $(\bowtie) \cdot$ W.-b. Chen • Q.-h. Qiu •

H. Li

Department of Radiology, Guangdong Academy of Medical

Sciences/Guangdong General Hospital,

No. 106 Zhongshan Er Road, 510080 Guangzhou, Guangdong Prov.,

People's Republic of China

e-mail: liang_changhong@126.com

Z.-p. Zhang

Applied Science Lab, GE Healthcare, GE Tower 10/F,

Huacheng Ave. 87, Tianhe District, 510623 Guangzhou,

Guangdong Prov., People's Republic of China
Key Points

- Intravoxel incoherent motion (IVIM) analysis permits separate quantification of diffusion and perfusion.

- IVIM DWI is a feasible technique for investigating primary NPC.

- IVIM suggests that primary NPC tissue voxels exhibit both perfusion and diffusion.

Keywords Intravoxel incoherent motion · MRI ·

Nasopharyngeal carcinoma $\cdot$ DWI $\cdot$ Enlarged adenoids
Abbreviations
ADC apparent diffusion coefficient
$D \quad$ pure molecular diffusion
$D^{*} \quad$ perfusion-related diffusion
$f \quad$ perfusion fraction
IVIM intravoxel incoherent motion
NPC nasopharyngeal carcinoma

\section{Introduction}

Worldwide, nasopharyngeal carcinoma (NPC) is a rare malignancy, but it shows marked geographic and racial variation in incidence [1] and is particularly endemic in southern China [2]. Other benign nasopharyngeal thickenings, such as enlarged adenoids, may also be detected in cross-sectional imaging, either incidentally or in patients examined specifically for ear, nose and throat symptoms [3]. Conventional MRI is the preferred imaging modality for the diagnosis, staging and monitoring of nasopharyngeal tumours owing to its superior soft tissue contrast resolution. Unfortunately, both conventional MRI and CT are limited in their abilities to differentiate between NPC and benign hyperplasia because of the overlapping signal characteristics and enhancement of these lesions following contrast administration [4]. 
Diffusion-weighted imaging (DWI) produces contrast based on differences in water diffusivity in distinct tissues. Because DWI can be performed relatively rapidly and is noninvasive, it is an attractive adjunct to anatomic MRI for the characterization of head and neck cancers. In recent years, several studies have demonstrated the utility of DWI for differentiating benign and malignant head and neck disease and for differentiating between histological types of certain malignancies [5-7]. However, some studies have shown that perfusion can substantially confound diffusion measurements because of the incoherent motion of blood in pseudorandom capillary networks at the macroscopic level [8]. Intravoxel incoherent motion (IVIM) analysis was developed to permit the separate quantification of diffusion and perfusion effects $[9,10]$. Blood perfusion is an important biomarker that differentiates NPC from benign nasopharyngeal thickening [11]; therefore, IVIM analysis may be more advantageous and sensitive than conventional diffusion imaging in characterizing NPC.

According to IVIM theory [8], signal attenuation as a function of the $b$ value may be expressed through a biexponential equation with three diffusion parameters: the perfusion fraction, $f$ (i.e. fractional volume occupied in the voxel by flowing spins); pure molecular diffusion, $D$; and perfusion-related diffusion, $D^{*}$ [12]. Pilot studies have suggested potential clinical applications for IVIM in head and neck cancers [13, 14], but to our knowledge, no published studies have evaluated the feasibility of performing IVIM at the primary site in NPC patients. Thus, the aim of this study was to compare the $D, D^{*}$ and $f$ values obtained using DW imaging based on the IVIM theory between patients with primary NPC and patients with enlarged adenoids.

\section{Materials and methods}

\section{Patient selection}

This prospective single-centre study was approved by the Research Ethics Committee of Guangdong Academy of Medical Sciences. Written informed consent was obtained from all patients and the caretakers on behalf of minors. From December 2011 to May 2013, 65 consecutive patients (48 men, 17 women; mean age, 51 years; age range, 16-69 years) underwent diagnostic or staging MRI evaluation and IVIM DWI of the primary site. These patients had suspected nasopharyngeal neoplasms and were admitted by an ENT (ear, nose and throat) doctor following examination with a disposable pharyngeal mirror or nasopharyngeal fibrescope. The NPC detection rates of MRI, endoscopy and biopsy were compared. The mean interval between MRI examination and subsequent nasopharyngeal biopsy was 3 days (range, 0-11 days). The initial study population comprised 40 patients with newly diagnosed NPC (29 men and 11 women; mean age, 55 years) and 25 patients with enlarged adenoids ( 19 men and 6 women; mean age, 33 years).

\section{MRI protocol}

MR imaging examinations of the whole nasopharynx were performed using a 3.0-T whole-body system (Signa Excite HD, GE Healthcare, Milwaukee, WI) with a 40-mT/m maximum gradient capability and a standard receive-only head and neck coil. The imaging protocol included axial T1weighted spin-echo images (repetition time (TR)/echo time (TE) $600 / 23 \mathrm{~ms}, 4 \mathrm{~mm}$ section thickness with a $1-\mathrm{mm}$ intersection gap and number of excitations (NEX) equal to 2), contrast-enhanced axial and coronal T1-weighted spin-echo images following a bolus injection of $0.1 \mathrm{mmol} / \mathrm{kg}$ of gadolinium diethylenetriaminepentaacetic acid (Gd-DTPA; Bayer Healthcare, Berlin, Germany), and axial T2-weighted turbo spin-echo images with fat suppression (TR/TE 5,200/137 ms; $4 \mathrm{~mm}$ section thickness, with $1 \mathrm{~mm}$ intersection gap and NEX=2) using a $512 \times 288$ imaging matrix.

\section{IVIM DW imaging sequence}

The IVIM DW imaging sequence was performed prior to the injection of Gd. Thirteen $b$ values $(0,10,20,30,50,80,100$, $150,200,300,400,600$ and $800 \mathrm{~s} / \mathrm{mm}^{2}$ ) were applied with a single-shot diffusion-weighted spin-echo echo-planar sequence. The lookup table of gradient directions was modified to allow multiple $b$ value measurements in one series. Parallel imaging was used with an acceleration factor of 2. A local shim box covering the nasopharynx region was applied to minimize susceptibility artefacts. In total, 14 axial slices covering the nasopharynx were obtained with a $24-\mathrm{cm}$ field of view, $4 \mathrm{~mm}$ slice thickness, $1 \mathrm{~mm}$ slice gap, 3,000 $\mathrm{ms}$ TR, $58 \mathrm{~ms}$ TE, $128 \times 128$ matrix and NEX=2. The nominal scan time was 3 min and $45 \mathrm{~s}$.

\section{IVIM DW MRI analysis}

According to IVIM, the signal intensities and $b$ values are related as follows:

$S_{b} / S_{0}=(1-f) \exp (-\mathrm{b} D)+f \exp \left(-\mathrm{b} D^{*}\right)$

where $S_{0}=$ signal intensity at the $b$ value of $0 ; S_{b}=$ signal intensity at the $b$ value denoted by the subscript; $D=$ true diffusion coefficient of a water molecule; $D^{*}=$ pseudo-diffusion coefficient due to microcirculation; and $f=$ microvascular volume fraction, indicating the fraction of diffusion related to microcirculation. Because $D^{*}$ is roughly one order of magnitude greater than $D[15],-b D^{*}$ would be less than -3 at a high 
$b$ value $\left(>200 \mathrm{~s} / \mathrm{mm}^{2}\right)$, and the term $f \exp \left(-\mathrm{b} D^{*}\right)$ would be less than $0.05 f$ and can therefore be neglected. In this case, Eq. (1) can be simplified as follows:

$$
\mathrm{S}_{b} / \mathrm{S}_{0}=(1-f) \exp (-\mathrm{b} D)
$$

Hence, for high $b$ values $\left(300,400,600\right.$ and $\left.800 \mathrm{~s} / \mathrm{mm}^{2}\right) \mathrm{S}_{b}$ was first fitted to Eq. (2) using a linear model, and the true diffusion coefficient $D$ was calculated. Although we had previously calculated the $f$ value, its accuracy was not acceptable; $f$ was recalculated using Eq. (1). Then, we fitted $\mathrm{S}_{b}$ for all $b$ values using Eq. (1) with a fixed $D$ value using the nonlinear Levenberg-Marquardt method. When fitting Eq. (1), the initial estimated values for $f$ and $D^{*}$ were set as the previously calculated $f$ value from Eq. (2) and $10 \times 10^{-3} \mathrm{~mm}^{2} / \mathrm{s}$, respectively. Subsequently, the $f$ and $D^{*}$ were obtained.

The ADC value was calculated by fitting $b_{0}$ image and DWIs at five non-zero $b$ values $(200,300,400,600$ and $800 \mathrm{~s} / \mathrm{mm}^{2}$ ) into the conventional ADC equation (Eq. (3)) [9].

$\mathrm{S}_{b} / \mathrm{S}_{0}=\exp (-b \mathrm{ADC})$

\section{Statistical analysis}

The mean values of all IVIM parameters were measured independently by two experienced radiologists in our department. A freehand ROI was positioned to cover as much nasopharyngeal tissue as possible while avoiding large vessels and areas of necrosis.

A nonparametric Mann-Whitney test was used to compare IVIM parameters between primary NPC and enlarged adenoid cases. The $D$ value (with independent significance between two groups) and ADC were assessed using a ROC curve to estimate the diagnostic tolerance. All analyses were performed using SPSS version 13.0 for Windows (SPSS, Chicago) and MedCalc (MedCalc Software, Acacialaan 22, B-8400 Ostend, Belgium). $P<0.05$ was considered significant.

\section{Inter-rater reliability}

Intra-class correlation coefficient (ICC) and BlandAltman (BA) analyses were performed to test interrater reliability. An ICC value close to 1 represented high reliability, and the difference between the two raters was considered insignificant if the $95 \%$ confidence interval of the difference included 0 .

\section{Results}

IVIM DWI was successful at the primary site in 60 of 65 patients (45 men and 15 women; mean age, 52 years; age range, 16-69 years), including 37 NPC cases and 23 enlarged adenoid cases confirmed by subsequent nasopharyngeal biopsy. IVIM DWI was unsuccessful in the remaining five patients because of susceptibility artefacts around the skull base and paranasal sinuses (three cases) or motion artefacts due to swallowing (two cases). Altogether, IVIM DWI failed in three cases of NPC and two cases of enlarged adenoids. The mean tumour volumes $( \pm \mathrm{SD})$ for patients with NPC and enlarged adenoids were $22.50 \pm 6.06$ and $8.52 \pm 2.78 \mathrm{~cm}^{3}$, respectively, according to the summation-of-areas technique.

The values of the IVIM parameters for cases of NPC and enlarged adenoids are presented in Table 1. Our data demonstrated that $D(P=0.001)$ and $f(P<0.0001)$ were significantly lower in patients with primary NPC than in patients with enlarged adenoids, whereas $D^{*}$ was significantly higher $(P<0.0001)$ in the NPC group. However, the difference in the ADC observed between the two groups did not reach significance $(P>0.05)$.

Box plots comparing $D, D^{*}$ and $f$ between patients with NPC and patients with enlarged adenoids are shown in Fig. 1. As shown in Fig. 2, the ROC analysis indicated that when both sensitivity and specificity were adjusted to produce the highest accuracy, the optimal $D$ and ADC thresholds for distinguishing primary NPC from enlarged adenoids were $0.75 \times 10^{-3} \mathrm{~mm}^{2} / \mathrm{s}$ and $0.936 \times 10^{-3} \mathrm{~mm}^{2} / \mathrm{s}$ respectively. Figure 2 shows that the AUC for $D(0.849)$ was significantly larger than $\mathrm{ADC}(0.566)(P<0.05)$.

IVIM DW images of NPC (Fig. 3) and enlarged adenoids (Fig. 4), which were performed with $13 b$ values (in the range 0-800 s/ $\mathrm{mm}^{2}$ ) are shown. The diffusion-weighted signal decayed in a bi-exponential manner within the range of low $b$ values (0-200 s/mm ${ }^{2}$ ). In addition, the signal fitting curves (Figs. 3c and 4c) appeared to differ between the patients with NPC and those with enlarged adenoids. The slope of the fitting curve in NPC patients was larger than that in patients with enlarged adenoids, especially within the range of low $b$ values $\left(0-200 \mathrm{~s} / \mathrm{mm}^{2}\right)$. This result demonstrates that the DWI signal was higher in the NPC group; thus, the value of $D$ was lower in patients with NPC than in patients with enlarged adenoids.

Table 1 IVIM parameters of NPC and enlarged adenoids

\begin{tabular}{lccc}
\hline Parameter & NPC $(n=37)$ & $\begin{array}{l}\text { Enlarged } \\
\text { adenoids }(n=23)\end{array}$ & $P$ value* \\
\hline$D^{*}\left(\times 10^{-3} \mathrm{~mm}^{2} / \mathrm{s}\right)$ & $152.96 \pm 27.41$ & $48.33 \pm 17.42$ & 0.000 \\
$D\left(\times 10^{-3} \mathrm{~mm}^{2} / \mathrm{s}\right)$ & $0.69 \pm 0.12$ & $0.78 \pm 0.06$ & 0.001 \\
$f(\%)$ & $16.44 \pm 2.01$ & $26.72 \pm 4.91$ & 0.000 \\
$\mathrm{ADC}\left(\times 10^{-3} \mathrm{~mm}^{2} / \mathrm{s}\right)$ & $1.10 \pm 0.13$ & $1.13 \pm 0.07$ & 0.543 \\
\hline
\end{tabular}

Unless otherwise indicated, data are means \pm standard deviations

* Mann-Whitney $U$ test for differences in IVIM parameters between nasopharyngeal carcinoma and nasopharyngeal hyperplasia 

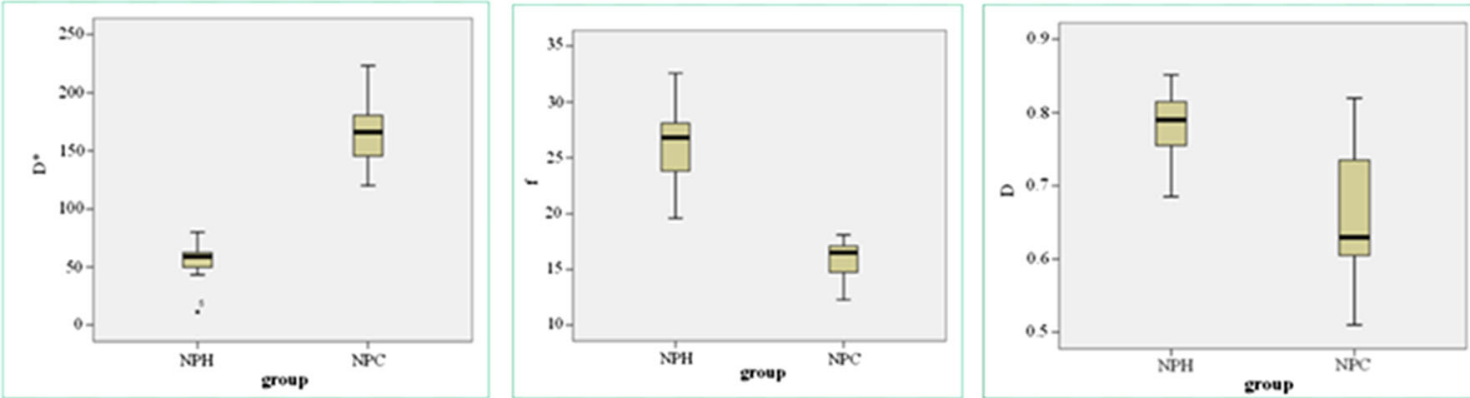

Fig. 1 Box plots of a $D^{*}, \mathbf{b} f$, and $\mathbf{c} D$ comparing patients with NPC and patients with enlarged adenoids (NPH). The top and bottom of the boxes indicate the first and third quartiles, respectively. The length of box

\section{Discussion}

The conventional ADC model assumes that diffusion-weighted signals should decay mono-exponentially with $b$ value. However, the blood flow in the capillary network contributes to the diffusion-weighted signal and decreases in a biexponential fashion at lower $b$ values $\left(<200 \mathrm{~s} / \mathrm{mm}^{2}\right)$. Because the IVIM model fits the signal decay with a bi-exponential decay, as opposed to the mono-exponential decay described by the ADC model, the IVIM parameters may reflect water diffusion and blood perfusion more accurately [8]. The IVIM model allows the estimation of four parameters $\left(D, f, D^{*}\right.$ and ADC) simultaneously; $f$ and $D^{*}$ are related to blood perfusion, and $D$ is related to water diffusion. The IVIM model has been used to investigate chronic brain ischaemia [8], human placental function [16], liver cirrhosis [9], muscle inflammatory myopathy [17] and renal perfusion [18]. Recently, this model was

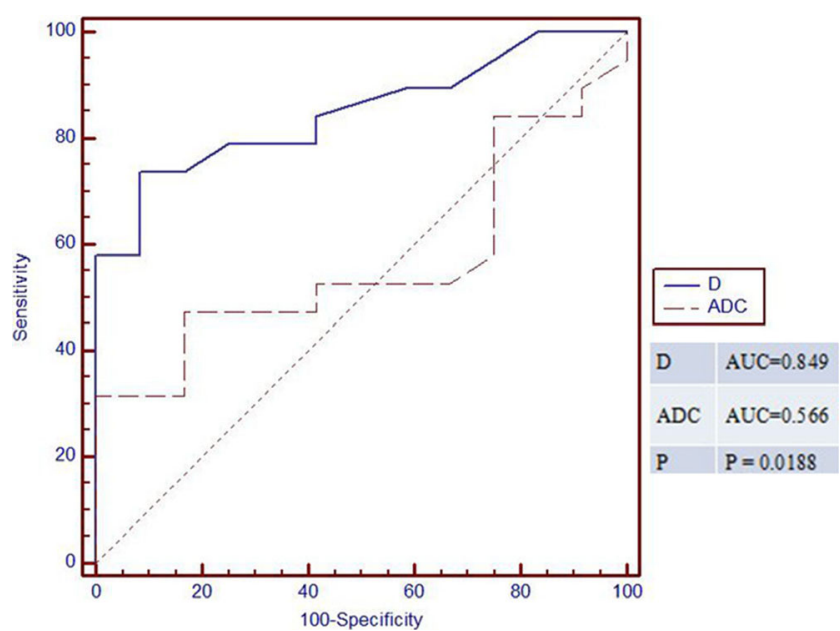

Fig. 2 Receiver operating characteristic (ROC) curve generated according to the $D$ and ADC value. The $D$ and ADC value cut-off points were determined to be $0.75 \times 10^{-3} \mathrm{~mm}^{2} / \mathrm{s}$ and $0.936 \times 10^{-3} \mathrm{~mm}^{2} / \mathrm{s}$ for nasopharyngeal disease. At a $D$ value of $0.75 \times 10^{-3} \mathrm{~mm}^{2} / \mathrm{s}$, the sensitivity and specificity for the prediction of NPC were 0.826 and 0.649 , respectively. At an ADC value of $0.936 \times 10^{-3} \mathrm{~mm}^{2} / \mathrm{s}$, the sensitivity and specificity for the prediction of NPC were 0.316 and 1 , respectively. The ROC curves demonstrated that $D$ had a higher AUC than ADC value $(P<0.05)$ represents the interquartile range, within which $50 \%$ of the values were located. The solid line within each box is the median. The error bars show the minimum and maximum values (range), with outliers indicated as dots

also expanded to characterize prostate [19] and breast [20] tumours. However, few studies have performed IVIM DW MRI of the head and neck $[13,14]$, and ours is one of the first to apply the IVIM model to primary NPC. In our study, IVIM DWI was performed successfully in the nasopharynx in $92.3 \%$ of cases, whereas the results of five cases were severely degraded because of susceptibility artefacts (three cases) and swallowing-related motion artefacts (two cases), consistent with the findings of a previous nasopharyngeal DWI study [7]. The use of local shim, parallel imaging and a reduced TE yielded high quality IVIM DW imaging in our study.

The use of the standard IVIM model may cause systematic over- or underestimation of diffusion parameters, depending on the underlying tissue architecture. For example, a recent study demonstrated that bi-exponential models for IVIM may be used to model the DW-MRI signal decay of tumour tissues, suggesting that most tumour tissue voxels exhibit both perfusion and diffusion and that diffusion in tumour tissues is either free or restricted [14]. Furthermore, the ADC models are too simple to account for the complex incoherent motions in tumours because they do not consider blood perfusion in tumour tissues. Therefore, Guiu et al. recently suggested that the ADC model should be abandoned [12] because it fits a biexponential decay with a mono-exponential equation, which is mathematically unacceptable except in cases when the $b$ values used are less affected by the perfusion process (i.e. $>200 \mathrm{~s} / \mathrm{mm}^{2}$ ). In such cases, the result of the ADC model simply corresponds to the calculation of $D$, which is the true water diffusion coefficient, reflecting both intra- and extracellular molecular diffusion. In our study, the diffusion-weighted signals at four $b$ values (300, 400, 600 and $800 \mathrm{~s} / \mathrm{mm}^{2}$ ) were fitted using a mono-exponential equation to calculate $D$, which, together with local shim, a short TE, parallel imaging and improved SNR at 3.0 T, may have allowed the calculation of a more reliable value for $D$. We found that $D$ was significantly lower in cases of primary NPC than in cases of enlarged adenoids (mean, $0.69 \times 10^{-3} \mathrm{~mm}^{2} / \mathrm{s}$ vs $0.78 \times 10^{-3} \mathrm{~mm}^{2} / \mathrm{s}$, respectively; $P=0.001$ ), indicating that $D$ was restricted in NPC cases. Simultaneously, the data suggest that IVIM DWI has greater clinical potential to distinguish two tumours on the 

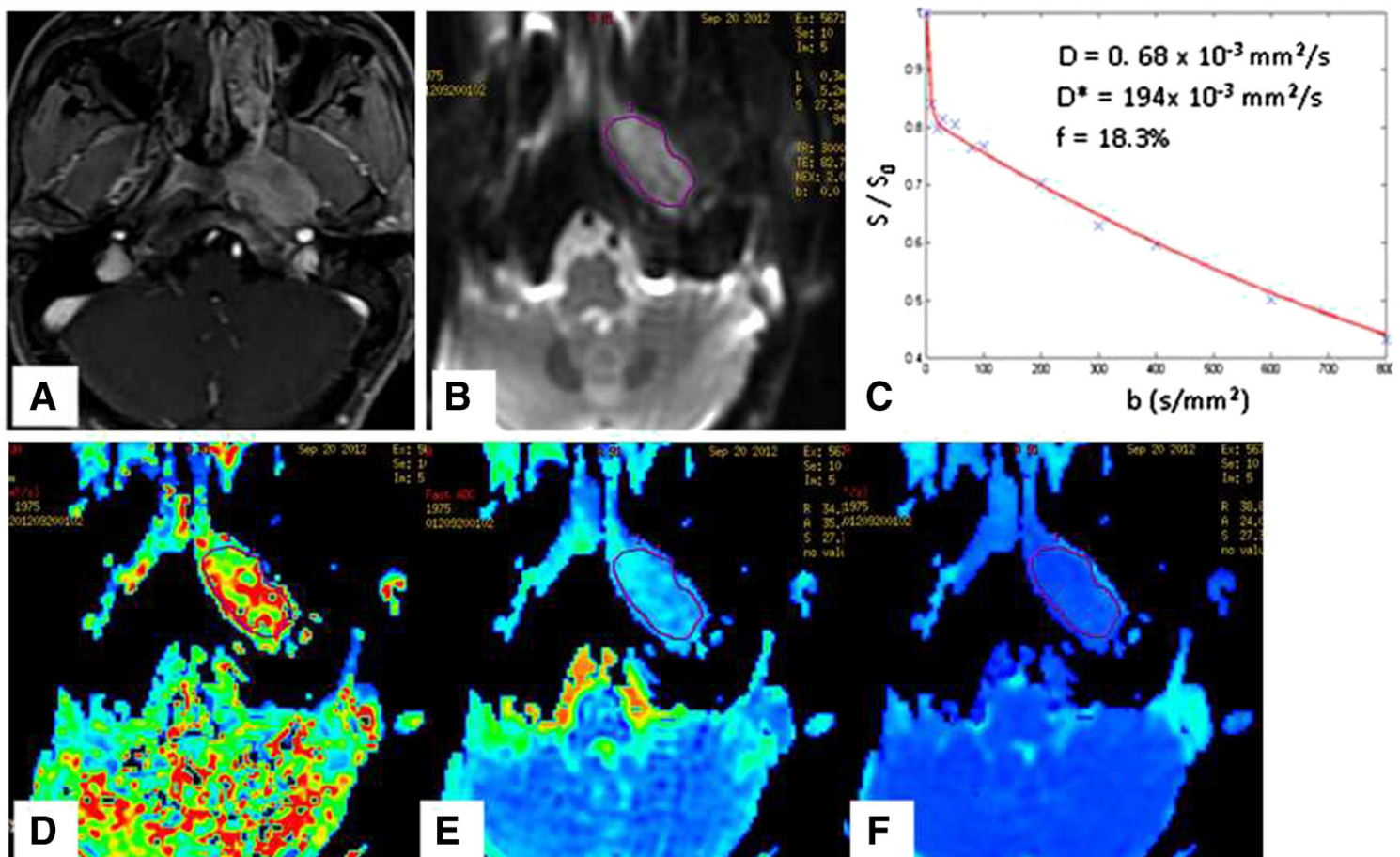

Fig. 3 IVIM DW images with $13 b$ values (in the range $0-800 \mathrm{~s} / \mathrm{mm}^{2}$ ) from a 35-year-old man referred to our department for confirmation of the NPC diagnosis. a Axial T1-weighted contrast-enhanced MR image and b DWI image. c Signals decayed bi-exponentially with $b$ value, as shown by the fitting curve (red line). With this distinctive signal decay curve

basis of their $D$ values with a threshold of $0.75 \times 10^{-3} \mathrm{~mm}^{2} / \mathrm{s}$. The significant differences in $D$ values between the two types of lesions investigated in the present study may be due to differences in NPC in terms of cellularity and extracellular matrix composition; the increased cellularity and varying amounts of stromal tissues in malignant tumours would reduce water diffusivity [21]. Despite the significant differences in the overall $D$ between primary NPC and enlarged adenoids, the $D$ values for NPC cases varied considerably and, importantly, overlapped appreciably between the two types of samples. In addition, Table 1 showed that the difference in the ADC between the NPC and enlarged adenoids did not reach significance $(P>0.05)$. Further, Fig. 2 demonstrated that the AUC for $D(0.849)$ was significantly larger than $\operatorname{ADC}(0.566)$ $(P<0.05)$. Therefore, we are confident that $D$ will be more useful than ADC in clinical practice to help differentiate NPC from benign nasopharyngeal hyperplasia in high-risk populations.

In IVIM theory (Eq. 1), $D^{*}$ is linked to perfusion, and perfusion levels may depend on tumour microvessel attenuation. Furthermore, the $D^{*}$ value is determined according to the signal intensity ratios of the blood capillaries. In our study, $D^{*}$ was more than 10 times greater than $D$, which explains why $D^{*}$ has a stronger influence on signal decay when $b$ is less than $200 \mathrm{~s} / \mathrm{mm}^{2}$. In addition, the accuracy of $D^{*}$ estimations improves with increased sampling in the $0-200 \mathrm{~s} / \mathrm{mm}^{2}$ range profiles, the unique diffusion- and perfusion-related parameters of this NPC patient were calculated and displayed. Within the range of low $b$ values $\left(0-200 \mathrm{~s} / \mathrm{mm}^{2}\right)$, this fitting curve demonstrates a large slope, meaning a low $D$ value. Parametric images enabled the presentation of the $\mathbf{d} D^{*}, \mathbf{e} f$ and $\mathbf{f} D$ parameters

[2-4]. Using an 11-b DW imaging sequence, Guiu et al. [22] reported that seven $b$ values $\left(0,5,15,25,35,50\right.$ and $\left.100 \mathrm{~s} / \mathrm{mm}^{2}\right)$ could be used to model the first portion of the bi-exponential decay curve, encompassing both $D$ and $D^{*}$. In our study, a 13- $b$ DW imaging sequence was used, and nine of these $b$ values were applied to model this region of the bi-exponential curve. The results showed a mean $D^{*}$ value for primary NPC that was in agreement with that of a previous study on metastatic nodes by $\mathrm{Lu}$ et al. (ranging from 76.18 to $180.39 \times 10^{-3} \mathrm{~mm}^{2} / \mathrm{s}$ ) [14]. Furthermore, we found a significant increase in $D^{*}$ for primary NPC (mean, $152.96 \times 10^{-3} \mathrm{~mm}^{2} / \mathrm{s}$ vs $48.33 \times 10^{-3} \mathrm{~mm}^{2} / \mathrm{s}$; $P<0.0001$ ), thereby confirming that $D^{*}$ is sensitive to differences in NPC perfusion. This increase may result from differences in the histologic structure of primary NPC, which has abundant vessels. Interestingly, the degree of MRI enhancement revealed increased capillary microcirculation in patients with primary NPC [21]. In the current study, the obtained $D^{*}$ value was significantly higher in primary NPC patients than in those with enlarged adenoids, suggesting that $D^{*}$ may be a biomarker for the diagnosis and differentiation of NPC.

In another recent study, the range of $f$ values estimated using the IVIM model for the neck metastatic node was 13$42 \%$ [14], similar to those in our study. Surprisingly, we found that $f$ was significantly decreased in primary NPC, although malignant tumours have been associated with 

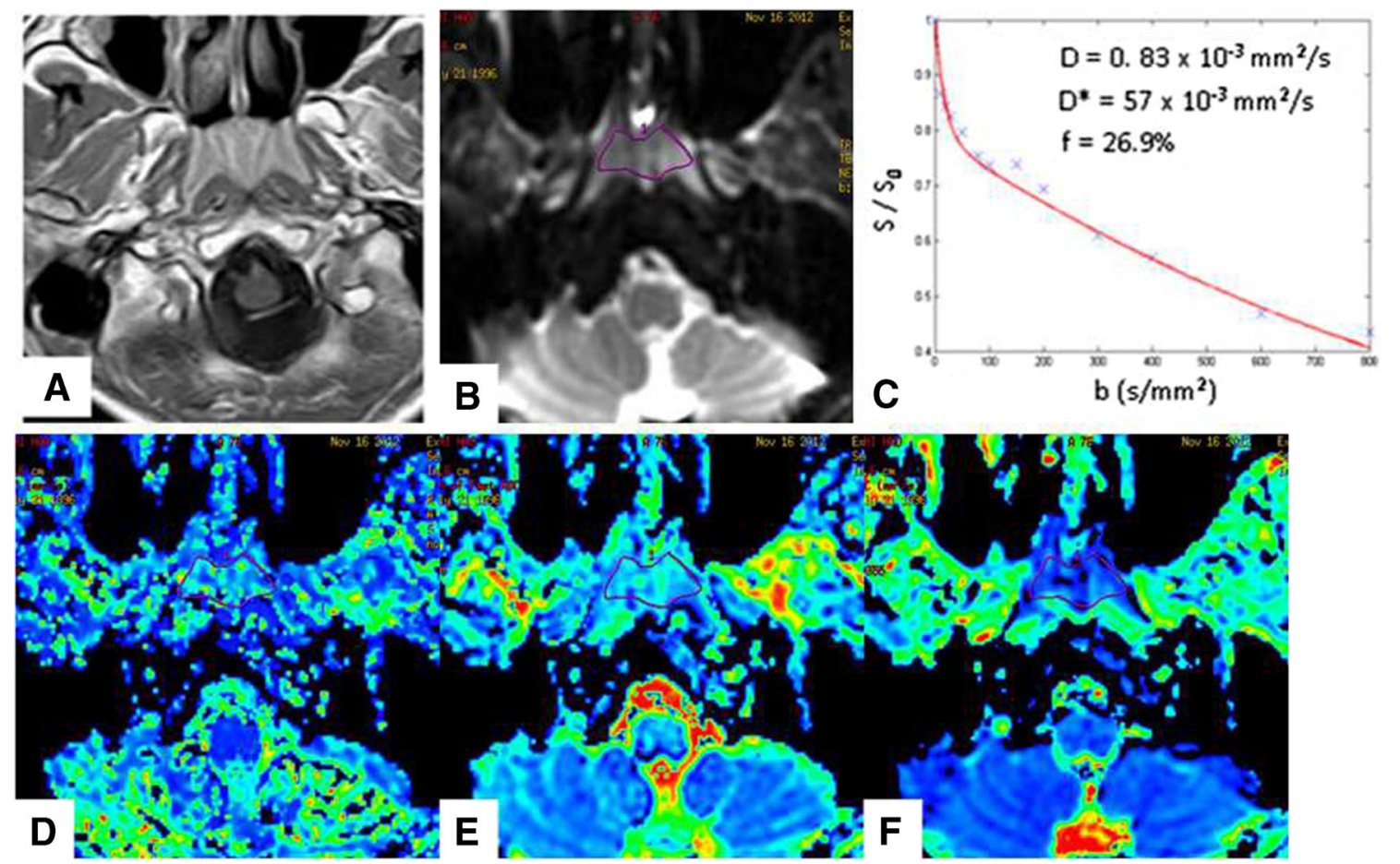

Fig. 4 IVIM DW images with $13 b$ values (in the range $0-800 \mathrm{~s} / \mathrm{mm}^{2}$ ) from a 19-year-old man referred to our department for confirmation of the enlarged adenoid diagnosis. a Axial T1-weighted contrast-enhanced MR image and b DWI image. c Signals decayed bi-exponentially with $b$ value, as shown by the fitting curve (red line). Within the range of low $b$ values (0-200 s/mm ${ }^{2}$ ), the slope of this distinctive signal decay curve was smaller, and indicate a high $\mathrm{D}$ value. The calculated diffusion- and perfusion-related parameters are also displayed. Parametric imaging enabled the presentation of the $\mathbf{d} D^{*}, \mathbf{e} f$ and $\mathbf{f} D$ parameters

increased capillary perfusion [23]. The same paradoxical evolution of $f$ was previously noted in studies using IVIM DW MRI of the fatty liver [22] and hepatocellular carcinoma [24]. One convincing explanation for this apparent paradox was provided by Lemke et al. [25], who clearly demonstrated that $f$ is dependent on TE; during longer TE, the signal decays further at low $b$ values, and $f$ increases. The TE-dependence of $f$ is most important for tissues with considerably shorter transversal relaxation times than blood. This effect would likely be significant for IVIM imaging of organs with short $\mathrm{T}_{2}$ times, such as primary NPC and potentially enlarged adenoids. However, in the IVIM approach, relaxation effects are neglected, which may be appropriate when the relaxation time of the tissue of interest is similar to that of blood. However, when these relaxation times diverge, e.g. in cases of NPC and enlarged adenoids, the extracted perfusion-related parameters may depend on measurement parameters such as TE and TR. Indeed, cases of NPC and enlarged adenoids demonstrated completely different $\mathrm{T}_{2}$ times. These differences may explain why $f$ values are greatly overestimated and require specific $\mathrm{T}_{2}$ corrections [25]. Unfortunately, we did not perform $\mathrm{T}_{2}$ calculations of NPC and enlarged adenoids (which are highly variable), and we were therefore unable to determine the "true" $f$ factor. The decrease in $f$ observed in primary NPC may be attributed to the decrease in the $T_{2}$ relaxation time of the tumour.
The current study had certain limitations. First, few cases of enlarged adenoids were included in the series. However, because benign hyperplasia and NPC exhibited a relatively narrow range and wide scattering of $D$ values, respectively, it is unlikely that the results of larger samples would be sufficiently different to affect our conclusions regarding the lack of a suitable $D$ threshold to differentiate NPC from enlarged adenoids. Secondly, IVIM DWI was unsuccessful in approximately $8.7 \%$ of cases because of severe image degradation resulting from magnetic field heterogeneities at the air-bone and air-soft tissue interfaces around the skull base and from physiological motion artefacts. This failure rate is similar to that reported previously [7]. Susceptibility artefacts increase with the use of higher $b$ values, typically approximately $1,000 \mathrm{~s} / \mathrm{mm}^{2}$. We tried to minimize this effect by setting the maximum $b$ value to $800 \mathrm{~s} / \mathrm{mm}^{2}$. Furthermore, the poor SNR at higher $b$ values may have decreased the accuracy of IVIM calculations.

In conclusion, $D$ and $f$ were significantly reduced in primary NPC compared with benign enlarged adenoids, possibly because of the restriction of water diffusion due to increased cellularity in malignant tumours. $D^{*}$ was significantly increased, which likely reflects the increased blood vessel generation and parenchymal perfusion in patients with primary NPC. These results demonstrate that the bi-exponential models of IVIM provide a reasonable model of DW-MRI 
signal decay in tissues and suggest that primary NPC tissue voxels exhibit both perfusion and diffusion.

Acknowledgments The scientific guarantor of this publication is Shui-xing Zhang. The authors of this manuscript declare relationships with the following companies: GE Healthcare. This study received funding from the National Scientific Foundation of China (grant 81171329) and grants from the Guangdong Science Foundation (grant S2011010000790). One of the authors has significant statistical expertise. Institutional review board approval was obtained. Written informed consent was obtained from all subjects (patients) in this study. Methodology: prospective, diagnostic or prognostic study, multicentre study.

Open Access This article is distributed under the terms of the Creative Commons Attribution Noncommercial License which permits any noncommercial use, distribution, and reproduction in any medium, provided the original author(s) and the source are credited.

\section{References}

1. Parkin DM, Bray F, Ferlay J et al (2005) Global cancer statistics. CA Cancer J Clin 55(2):74-108

2. Parkin DM, Whelan SL, Ferlay J et al (2002) Cancer incidence in five continents. VIII. IARC scientific publications no. 155. IARC, Lyon

3. King AD, Vlantis AC, Tsang RK et al (2006) Magnetic resonance imaging for the detection of nasopharyngeal carcinoma. AJNR Am J Neuroradiol 27(6):1288-1291

4. Vogl T, Dresel S, Schedel H et al (1989) MRI of the nasopharynx with Gd-DTPA: its value and differential diagnostic criteria. Röfo 150(5):516-522

5. Sumi M, Sakihama N, Sumi T et al (2003) Discrimination of metastatic cervical lymph nodes with diffusion-weighted MR imaging in patients with head and neck cancer. AJNR Am J Neuroradiol 24(8): 1627-1634

6. Razek AA, Megahed AS, Denewer A et al (2008) Role of diffusionweighted magnetic resonance imaging in differentiation between the viable and necrotic parts of head and neck tumors. Acta Radiol 49(3): 364-370

7. King AD, Ahuja AT, Yeung DK et al (2007) Malignant cervical lymphadenopathy: diagnostic accuracy of diffusion-weighted MR imaging. Radiology 245(3):806-813

8. Le Bihan D, Breton E, Lallemand D et al (1988) Separation of diffusion and perfusion in intravoxel incoherent motion MR imaging. Radiology 168(2):497-505

9. Luciani A, Vignaud A, Cavet $M$ et al (2008) Liver cirrhosis: intravoxel incoherent motion MR imaging - pilot study. Radiology 249(3):891-899
10. Patel J, Sigmund EE, Rusinek H et al (2010) Diagnosis of cirrhosis with intravoxel incoherent motion diffusion MRI and dynamic contrast-enhanced MRI alone and in combination: preliminary experience. J Magn Reson Imaging 31(3):589-600

11. Jin G, Su D, Liu L et al (2011) The accuracy of computed tomographic perfusion in detecting recurrent nasopharyngeal carcinoma after radiation therapy. J Comput Assist Tomogr 35(1):26-30

12. Guiu B, Cercueil JP (2011) Liver diffusion-weighted MR imaging: the tower of Babel? Eur Radiol 21(3):463-467

13. Sumi M, Nakamura T (2013) Head and neck tumors: assessment of perfusion-related parameters and diffusion coefficients based on the intravoxel incoherent motion model. AJNR Am J Neuroradiol 34(2): $410-416$

14. Lu Y, Jansen JF, Mazaheri Y et al (2012) Extension of the intravoxel incoherent motion model to non-gaussian diffusion in head and neck cancer. J Magn Reson Imaging 36(5):1088-1096

15. Le Bihan D (2008) Intravoxel incoherent motion perfusion MR imaging: a wake-up call. Editorial Radiol 249(3):748-752

16. Moore RJ, Issa B, Tokarczuk P et al (2000) In vivo intravoxel incoherent motion measurements in the human placenta using echo-planar imaging at 0.5 T. Magn Reson Med 43(2):295-302

17. Qi J, Olsen NJ, Price RR et al (2008) Diffusion-weighted imaging of inflammatory myopathies: polymyositis and dermatomyositis. J Magn Reson Imaging 27(1):212-217

18. Muller MF, Prasad PV, Edelman RR (1998) Can the IVIM model be used for renal perfusion imaging? Eur J Radiol 26(3):297-303

19. Riches SF, Hawtin K, Charles-Edwards EM et al (2009) Diffusionweighted imaging of the prostate and rectal wall: comparison of biexponential and monoexponential modelled diffusion and associated perfusion coefficients. NMR Biomed 22(3):318-325

20. Sigmund EE, Cho GY, Kim S et al (2011) Intravoxel incoherent motion imaging of tumor microenvironment in locally advanced breast cancer. Magn Reson Med 65(5):1437-1447

21. White ML, Zhang Y, Robinson RA (2006) Evaluating tumors and tumorlike lesions of the nasal cavity, the paranasal sinuses, and the adjacent skull base with diffusion-weighted MRI. J Comput Assist Tomogr 30(3):490-495

22. Guiu B, Petit JM, Capitan Vet al (2012) Intravoxel incoherent motion diffusion-weighted imaging in nonalcoholic fatty liver disease: a 3.0T MR study. Radiology 265(1):96-103

23. Lu CH, Yang CY, Wang CP et al (2010) Imaging of nasopharyngeal inflammatory pseudotumors: differential from nasopharyngeal carcinoma. Br J Radiol 83(985):8-16

24. Lewin M, Fartoux L, Vignaud A et al (2011) The diffusion-weighted imaging perfusion fraction $\mathrm{f}$ is a potential marker of sorafenib treatment in advanced hepatocellular carcinoma: a pilot study. Eur Radiol 21(2):281-290

25. Lemke A, Laun FB, Simon D et al (2010) An in vivo verification of the intravoxel incoherent motion effect in diffusion-weighted imaging of the abdomen. Magn Reson Med 64(6):1580-1585 